\title{
An LSTM based Kalman Filter for Spatio-temporal Ocean Currents Assimilation
}

\author{
Ziqiao Zhang \\ Electrical and Computer Engineering, Georgia Institute of \\ Technology \\ Atlanta, GA, US \\ ziqiao.zhang@gatech.edu \\ Fumin Zhang \\ Electrical and Computer Engineering, Georgia Institute of \\ Technology \\ Atlanta, GA, US \\ fumin@gatech.edu
}

\author{
Mengxue Hou \\ Electrical and Computer Engineering, Georgia Institute of \\ Technology \\ Atlanta, GA, US \\ mhou30@gatech.edu \\ Catherine R. Edwards \\ Skidaway Institute of Oceanography, Department of \\ Marine Sciences, University of Georgia \\ Savannah, GA, US \\ catherine.edwards@skio.uga.edu
}

\begin{abstract}
In this paper, we present a Long Short-Term Memory (LSTM)-based Kalman Filter for data assimilation of a 2D spatio-temporally varying depth-averaged ocean flow field for underwater glider path planning. The data source to the filter combines both the Eulerian flow map with the Lagrangian mobile sensor data stream. The depth-averaged flow is modeled as two components, the tidal and the non-tidal flow component. The tidal flow is modeled with $\mathrm{AD}$ CIRC (Advanced Three-Dimensional Circulation Model), while the non-tidal flow field is modeled by a set of spatial basis functions and their time series coefficients. The spatial basis functions are the principal modes derived by performing EOF (Empirical Orthogonal Functions) analysis on the historical surface flow field measured by high frequency radar (HFR), and the temporal coefficients of the spatial basis function are modeled by an LSTM neural network. The Kalman Filter is performed to combine the dynamics derived from the LSTM network, and the observations from the glider flow estimation data. Simulation results demonstrate that the proposed data assimilation method can give flow field prediction of reasonable accuracy.
\end{abstract}

\section{CCS CONCEPTS}

- Computing methodologies $\rightarrow$ Modeling and simulation.

\section{KEYWORDS}

data assimilation, LSTM, ocean flow field

ACM Reference Format:

Ziqiao Zhang, Mengxue Hou, Fumin Zhang, and Catherine R. Edwards. 2019. An LSTM based Kalman Filter for Spatio-temporal Ocean Currents

Permission to make digital or hard copies of all or part of this work for personal or classroom use is granted without fee provided that copies are not made or distributed for profit or commercial advantage and that copies bear this notice and the full citation on the first page. Copyrights for components of this work owned by others than ACM must be honored. Abstracting with credit is permitted. To copy otherwise, or republish, to post on servers or to redistribute to lists, requires prior specific permission and/or a fee. Request permissions from permissions@acm.org.

WUWNET'19, October 23-25, 2019, Atlanta, GA, USA

(C) 2019 Association for Computing Machinery.

ACM ISBN 978-1-4503-7740-9/19/10 . \$ \$15.00

https://doi.org/10.1145/3366486.3366522
Assimilation. In WUWNET'19: International Conference on Underwater Networks Systems (WUWNET'19), October 23-25, 2019, Atlanta, GA, USA. ACM, New York, NY, USA, 7 pages. https://doi.org/10.1145/3366486.3366522

\section{INTRODUCTION}

Underwater gliders are moving robotic sensing platforms [15] that are able to perform persistent surveying missions in the ocean for data collection $[11,20,28]$. They take advantage of buoyancy and attitude to move through the water column [6, 23, 25, 27], surfacing at defined intervals to communicate with the onshore dockserver to receive updated mission commands and waypoint lists. Since the forward speed of underwater gliders is relatively low compared to flow speed, ocean flow could have critical influence on glider's motion. Therefore, flow field prediction with satisfactory accuracy and resolution is a fundamental requirement for underwater glider path planning.

In general, there are two kinds of data sources from which flow field information can be obtained: through direct measurement, and from ocean circulation models. Depending on the area of interest, direct measurements of flow can be made in real or near-real time from cabled acoustic doppler current profilers (ADCPs) or at the surface from high frequency radar (HFR). ADCPs are typically moored or installed onto buoys or ships to measure ocean current as a function of depth, but cannot provide spatial variability of the field over long time series. HFR systems provide estimates of surface flow data over large areas in coastal regions that can be covered by long- or short-range radar, but glider navigation requires accurate estimates of depth-averaged flow, which is not necessarily represented by surface measurements. Gliders navigate by dead-reckoning, based on the depth-averaged estimate of flow over its three-dimensional path, but these estimates of velocity are phase-lagged with respect to the surfacing interval, and cannot account for the spatial and temporal variation of the flow field. Ocean circulation models (e.g., the Regional Ocean Modeling System (ROMS) [22], the Terrain-following Ocean Modeling System (TOMS) [8], and the Hybrid Coordinate Ocean Model (HYCOM) [3]) can provide flow information over a large spatial domain, and forecast over several days, and have been applied extensively to navigation tasks of underwater gliders $[24,26]$. However, models 
are limited by their spatial and temporal resolution, and may contain uncertainty and error due to incomplete physics or boundary conditions, particularly in areas of large gradients [7, 9].

Data assimilation can be defined as the incorporation of observations into a dynamical model to improve forecasts. Observations of the system can be either Lagrangian data stream collected by a moving vehicle along its trajectory, or Eulerian map collected by static sensor networks. The model can be described by a set of differential equations derived either from geophysical models or through data-driven methods. Data assimilation methods can be divided into two classes, variational and sequential methods [1]. Variational methods compute the unknown parameters of the system by minimizing a cost function describing the misfit between the model and observation [5, 12]. Sequential methods takes a probabilistic framework, and propagate the system states estimation at each time step, given the dynamical system model and the observation information $[1,17]$.

In this work, we will adopt the sequential data assimilation method to provide short term predictions for glider navigation, decomposing and parameterizing spatial and temporal variability separately to save computation cost [2]. Empirical orthogonal function (EOF) analysis [14] is a well known method for data compression in two or more dimensions. With EOF analysis, the spatio-temporally varying flow field can be decomposed into a set of spatial basis functions that vary only in space, and a set of temporal coefficients that vary in time. The combination of spatial basis function and temporal coefficients can fully represent the spatial and temporal variability of the field. In this case, the temporal variation of the system can be described by the variation of the set of temporal coefficients, which can be modeled by the time-series analysis modeling techniques.

Obtaining correct transition and measurement models is crucial to achieve improved filtering accuracy. Various methods have been applied to model the transition and measurement model, and also to estimate the unknown state and measurement noise covariance. To obtain the transition and observation model, Neural Network based approaches have emerged in constructing temporal filters for dynamical systems. [4] presents a Long Short-Term Memory (LSTM) -based Kalman Filter method to model the human motion. [18] introduces a Neural Network-based Ensemble Kalman Filter method to interpolate sea surface temperature field. Noise covariance has been estimated making the assumption that the noise covariance is time-invariant, and applying Maximum Likelihood Estimation, Covariance-Matching Techniques, and other algorithms [16]. Other methods allow the noise covariance matrix to be time-varying $[13,21]$, and dynamically weights and predicts the evolution of states based on the transition model and the measurements.

In this work, we propose to develop a data assimilation method utilizing LSTM-based Kalman Filter that provides prediction of the depth-averaged ocean currents in a domain, combining the Eulerian flow map with the Lagrangian data stream. The depth-averaged flow is modeled as two components, the tidal and the non-tidal flow component. The tidal flow is modeled with ADCIRC (Advanced Three-dimensional Circulation Model) [19], while the non-tidal flow field is modeled by a set of spatial basis functions and their time series coefficients. The spatial basis functions are the principal modes derived by performing EOF analysis on the historical HFR flow field, and the temporal coefficients of the spatial basis function are modeled by LSTM network. We verify the proposed data assimilation method by comparing the simulated flow with HFR system measurements and observed flow during a glider deployment.

\section{PROBLEM FORMULATION}

In this section, we will first present the formulation of the problem, and then briefly describe the EOF analysis and the structure of LSTM network.

Consider a space-time flow field. Noisy observations of this flow field are taken at each time step in some time interval $\mathcal{T}$. We denote the set of observations at time step $t$ as $\mathbf{y}_{t} \in \mathbb{R}^{n}$. We assume that the physical process of this field can be described by the evolution of some unknown hidden states $\mathbf{x} \in \mathbb{R}^{m}$,

$$
\mathbf{y}_{t}=\mathbf{H}\left(\mathbf{x}_{t}\right)+\eta_{t},
$$

where $\mathbf{H}: \mathbb{R}^{m} \rightarrow \mathbb{R}^{n}$ describes the relationship between the hidden states and the observations. $\eta_{t} \in \mathbb{R}^{n}$ represents an independently and identically distributed Gaussian noise.

The dynamics of the hidden states is usually described by a set of differential equations

$$
\mathbf{x}_{t}=\mathbf{f}\left(\mathbf{x}_{t-1}\right)+n_{t}
$$

where $\mathrm{f}: \mathbb{R}^{m} \rightarrow \mathbb{R}^{m}$ denotes the dynamical model governing the evolution of the hidden states. $n_{t} \in \mathbb{R}^{m}$ represents an independently and identically distributed Gaussian noise.

The goal of this paper is to combine the Eulerian flow observation with the Lagrangian flow observation, in order to obtain a less noisy reconstructed flow field. From the probabilistic point of view, this problem can be formulated as a Bayesian filtering problem. Under certain assumptions, the Bayesian filtering problem can be solved by the famous Kalman Filter.

\subsection{EOF Analysis}

The objective of EOF analysis is to determine a set of complete and orthogonal functions that can characterize the spatial structures of a domain, and also the corresponding temporal variability of the spatial structures.

Denote a discrete space-time flow field as $Z$. The value of the field at time step $t \in \mathcal{T}$ and grid point $s \in \mathcal{S}$ is noted as the $t^{t h}$ row and $s^{t h}$ column element of matrix $Z$, noted by $Z(t, s)$. The covariance matrix $C$ of $Z$ is given by

$$
C=\frac{(Z-\mathbf{1} \bar{z})^{T}(Z-\mathbf{1} \bar{z})}{n-1},
$$

where $\bar{z}$ is a row vector representing the time-averaged flow speed at each grid point in the domain $\mathcal{S}, \bar{z}(s)$ denotes the time-averaged flow speed at the $s^{t h}$ grid point. 1 represents a column vector containing all one elements. EOF aims to find the linear combination of grid points that explains the maximum variance. The $k^{t h}$ EOF mode, denoted as $v_{k}$, is the eigenvector corresponding to the $k^{t h}$ largest eigenvalue of the covariance matrix. The time coefficient corresponding to the $k^{t h}$ EOF mode, denoted as $\beta_{k}$ is

$$
\beta_{k}(t)=\sum_{s=1}^{S}(Z(t, s)-\bar{z}(s)) v_{k}(s) .
$$




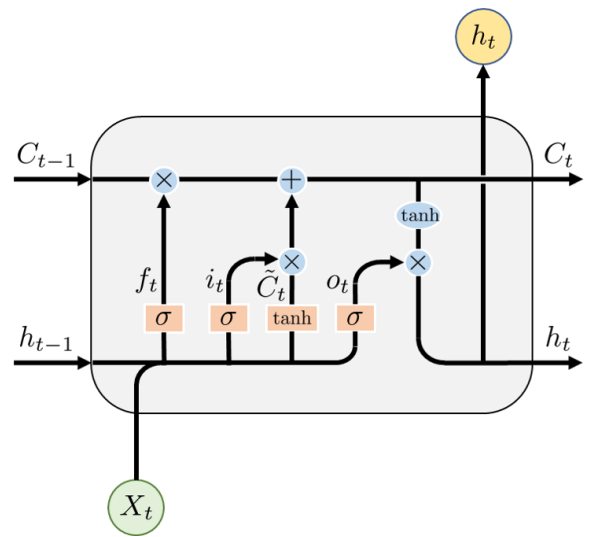

Figure 1: Structure of the LSTM block

The spatial and temporal varying flow field can be decomposed into the time-averaged field $\bar{z}(s)$, and the EOF modes $v_{k}(s)$ multiplied with time coefficients $\beta_{k}(t), Z(t, s)=\sum_{k=1}^{K} \beta_{k}(t) v_{k}(s)+\bar{z}(s)$. To make it more convenient to represent the flow field, we reshape $Z(t, s)$ into a $3 \mathrm{D}$ matrix $\xi(t, \mathbf{r})$, where $\mathbf{r}$ is a $2 \mathrm{D}$ column vector representing the $x, y$ position of a grid cell in the domain $\mathcal{S}$. In this work, we use $\xi$ to denote the flow velocity of each grid cell of the domain in either N/S or E/W direction. Similarly, each of the EOF modes, $v_{k}(s)$ is reshaped into a $2 \mathrm{D}$ matrix $\phi_{k}(\mathbf{r})$, and the averaged field, $\bar{z}(s)$ will be reshaped into a $2 \mathrm{D}$ matrix $\bar{\xi}(\mathbf{r})$. Then, the flow field can be represented as

$$
\xi(t, \mathbf{r})=\sum_{k=1}^{K} \beta_{k}(t) \phi_{k}(\mathbf{r})+\bar{\xi}(\mathbf{r})
$$

\subsection{LSTM}

The long short-term memory (LSTM) [10] method is a variant of a recurrent neural network, and is designed to address the problem of vanishing and exploding gradients faced by recurrent neural networks. By applying LSTM, we are able to build not only the short term relationship, but also long term dependencies in sequential data.

The equations for a general LSTM block are as follows,

$$
\begin{aligned}
& f_{t}=\sigma\left(W_{x f} x_{t}+W_{h f} h_{t-1}+b_{f}\right) \\
& i_{t}=\sigma\left(W_{x i} x_{t}+W_{h i} h_{t-1}+b_{i}\right) \\
& o_{t}=\sigma\left(W_{x o} x_{k}+W_{h o} h_{t-1}+b_{o}\right) \\
& \tilde{C}_{t}=\tanh \left(W_{x} \tilde{C}^{x_{t}}+W_{h \tilde{C}} h_{t-1}+b_{\tilde{C}}\right) \\
& C_{t}=f_{t} \circ C_{t-1}+i_{t} \circ \tilde{C}_{t-1} \\
& h_{t}=o_{t} \circ \tanh \left(C_{t}\right) \text {, }
\end{aligned}
$$

where $\sigma(\cdot)$ denotes the sigmoid function and $\circ$ denotes elementwise multiplication. As shown in Figure 1, $x_{t}$ is the input for the current LSTM block at time $t, h_{t-1}$ and $h_{t}$ are hidden states at time $t-1$ and $t$ respectively, and $C_{t-1}$ and $C_{t}$ are cell states. $f_{t}, i_{t}$ and $o_{t}$ are outputs of forget gate layer, input gate layer and output gate layer respectively.
The forget gate decides how much information from previous steps should be kept. The input gate decides how much input information will be used in the cell state. After running the first two gates, the cell state is also updated. The output gate decides how much information from the cell state will go to the hidden state. The input gate helps connect current input with the cell state which builds the short-term dependency. The forget gate relates hidden state from previous step to the current cell state and the output gate relates hidden state with cell state, which builds the longterm dependency. The weight matrices in Eq. (6) can be learned by LSTM networks through the training process given inputs and corresponding outputs. Then the LSTM networks with learned weight matrices are capable to model the dynamics between the input and the output in the sense of long and short term dependencies.

Using LSTM network does not require prior knowledge on the structure of the system. During the training process, we only need input data and the corresponding output data to train the network. After the network is trained, input data are required to run the LSTM network.

\section{DATA ASSIMILATION USING LSTM BASED KALMAN FILTER}

In this section, the proposed data assimilation method using LSTM based Kalman Filter is introduced. We will first introduce deriving the state transition model using the LSTM network. Then the data assimilation technique using LSTM based Kalman Filter will be introduced.

\subsection{State dynamics}

We assume that the dynamics model for the temporal coefficients can be written as follows

$$
B(t)=f(B(t-1), \ldots, B(t-p), t),
$$

where $B(t)=\left[\beta_{1}(t), \ldots, \beta_{K}(t)\right]^{T}, f(\cdot)$ is an unknown time-varying function and $p$ is the length of time window that describes its time dependency.

Since there is no deterministic model for the dynamics of temporal coefficients of the EOF modes, we consider applying LSTM to model the temporal variation of the flow field. Specifically, an LSTM network is used to model the dynamics of the temporal coefficients of the EOF modes,

$$
B(t)=g_{L S T M}(B(t-1), \ldots, B(t-p)),
$$

where $g_{L S T M}(\cdot)$ represents the trained LSTM-based Neural Network model of the temporal coefficients. By applying LSTM to model the dynamics of temporal coefficients, we are able to build the long short-term relationship between current coefficients and those from previous time steps.

The training process of the LSTM network is shown in Figure 2. First the EOF analysis is performed to derive the temporal coefficients of the historical ocean surface flow data. Then a time delay function is employed to generate the $N$ steps time-delayed vector $\mathbf{B}(t-1)=\left[B(t-1)^{T}, \ldots, B(t-N)^{T}\right]^{T} . \mathbf{B}(t-1)$ is the training input data of the LSTM network, and the corresponding output data of the LSTM network is $B(t)$. 


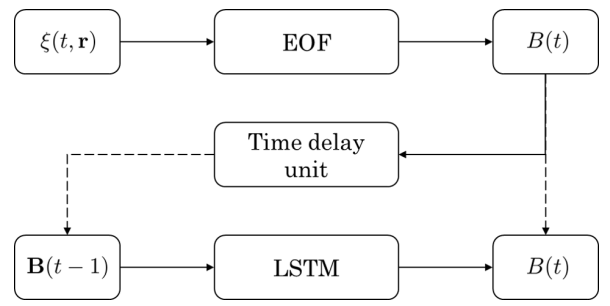

Figure 2: LSTM training for state equation

The Jacobian matrix of the state equation is needed to propagate the covariance matrix of the Kalman Filter at each iteration. We calculate the Jacobian matrix by linearizing $g_{L S T M}$ over a certain time window $T_{L}$. The following optimization problem is solved in each time window $T_{L}$ for a linearized model of the temporal coefficients,

$$
\min _{F_{k}, c_{k}} \sum_{\tau \in\left[t-T_{L}, t\right]}\left\|\beta_{k}(\tau)-F_{k}(\tau-1) \beta_{k}(\tau-1)-c_{k}\right\|
$$

The process update equations of the Kalman Filter can then be written as

$$
\begin{aligned}
& B^{(-)}(t)=g_{L S T M}(\mathbf{B}(t-1)) \\
& P^{(-)}(t)=F(t-1) P(t-1) F(t-1)^{T}+W(t-1),
\end{aligned}
$$

where $F(t-1)$ is a diagonal matrix whose $1^{s t}, \ldots, K^{t h}$ diagonal elements are $F_{1}(t-1), \ldots, F_{K}(t-1), W(t-1)$ is the variance of the process noise.

\subsection{Flow observation}

In this work, ocean currents are modeled as two components, the tidal component and the non-tidal component. We employ the predictive ocean model ADCIRC for modeling depth-averaged tidal currents. The non-tidal component is modeled by a set of spatial basis function and a set of time series coefficients correspondingly. Then according to Eq. (5), the surface flow at grid point $\mathbf{r}$ and time $t$ can be denoted as

$\xi^{s u r f}(t, \mathbf{r})=\sum_{k=1}^{K} \beta_{k}(t) \phi_{k}(\mathbf{r})+\bar{\xi}_{\text {non-tidal }}(\mathbf{r})+\xi_{\text {tidal }}(t, \mathbf{r})+n^{\text {surf }}$,

where the EOF modes $\phi_{k}(\mathbf{r})$, and the weights of the EOF modes, $\beta_{k}(t)$ are defined in Eq. (5).

Since the depth-averaged flow that glider experiences over each subsurface interval might be different from the surface flow that HFR measured, we assume the depth-averaged non-tidal flow can be approximated by the surface non-tidal flow multiplied with an unknown parameter $G \in \mathbb{R}$. Then the depth-averaged flow at grid point $\mathbf{r}$ and time $t$ can be modeled as

$\xi^{d e p t h}(t, \mathbf{r})=G\left(\sum_{k=1}^{K} \beta_{k}(t) \phi_{k}(\mathbf{r})+\bar{\xi}_{n o n-t i d a l}(\mathbf{r})\right)+\xi_{t i d a l}(t, \mathbf{r})+n^{\text {depth }}$
We combine the surface flow and the depth-averaged flow into a column vector and rewrite Eq. (11) (12) as follows,

$$
\begin{aligned}
z(t, \mathbf{r})=\left[\begin{array}{c}
\xi^{\text {surf }} \\
\xi^{\text {depth }}
\end{array}\right](t, \mathbf{r})= & {\left[\begin{array}{l}
1 \\
G
\end{array}\right]\left(\sum_{k=1}^{K} \beta_{k}(t) \phi_{k}(\mathbf{r})+\bar{\xi}_{\text {non-tidal }}(\mathbf{r})\right) } \\
& +\left[\begin{array}{c}
\xi_{\text {tidal }} \\
\xi_{\text {tidal }}
\end{array}\right](t, \mathbf{r})+\left[\begin{array}{c}
n^{\text {surf }} \\
n^{\text {depth }}
\end{array}\right] \\
= & H(t, \mathbf{r}) B(t)+M(t, \mathbf{r})+\left[\begin{array}{c}
n_{p}^{\text {surf }} \\
\text { depth } \\
n_{p}
\end{array}\right],
\end{aligned}
$$

where $H(t, \mathbf{r})=\left[\begin{array}{l}1 \\ G\end{array}\right]\left[\phi_{1}(\mathbf{r}), \ldots, \phi_{K}(\mathbf{r})\right]$,

and $M(t, \mathbf{r})=\left[\begin{array}{l}1 \\ G\end{array}\right] \bar{\xi}_{\text {non-tidal }}(\mathbf{r})+\left[\begin{array}{l}1 \\ 1\end{array}\right] \xi_{\text {tidal }}(t, \mathbf{r})$.

\subsection{Data Assimilation}

The parameter $G$ in Eq. (13) is unknown. Thus, we have a nonlinear observation equation, leading to a nonlinear filtering problem. To solve this nonlinear filtering problem, we decouple the state estimation and parameter estimation, and decompose a nonlinear filter into two sub-filters. Upon each surfacing event, the first sub-filter will derive state estimation with prior estimated $G$. Then given the updated state estimation, the second sub-filter will update estimation of the unknown parameter $G$ from glider derived flow estimate.

For the first sub-filter for state estimation, the filtering equations are given by

$$
\begin{aligned}
B^{(-)}(t) & =g_{L S T M}(\mathbf{B}(t-1)) \\
P^{(-)}(t) & =F(t-1) P(t-1) F(t-1)^{T}+W(t-1) \\
K(t) & =P^{(-)}(t) H(t, \mathbf{r})^{T}\left(H(t, \mathbf{r}) P^{(-)}(t) H(t, \mathbf{r})^{T}+R(t)\right)^{-1} \\
B(t) & =B^{(-)}(t)+K(t)\left(z(t, \mathbf{r})-H(t, \mathbf{r}) B^{(-)}(t)-M(t, \mathbf{r})\right) \\
P(t) & =P^{(-)}(t)-K(t) H(t, \mathbf{r}) P^{(-)}(t) .
\end{aligned}
$$

For the sub-filter of parameter estimation, we fix the states and estimate the parameter $G$. At each surfacing event, the state and observation equation are given by

$$
\begin{gathered}
G(t)=G(t-1)+w^{G}(t-1), \\
z^{\text {depth }}(t, \mathbf{r})=H^{G}(t, \mathbf{r}) G(t)+M^{G}(t, \mathbf{r})+n^{\text {depth }},
\end{gathered}
$$

where $w^{G}$ is the Gaussian distributed process noise with mean zero and variance $W^{G}, n^{\text {depth }}$ is the Gaussian distributed measurement noise with mean zero and variance $R^{G}, M^{G}(t, \mathbf{r})=\xi_{\text {tidal }}(t, \mathbf{r})$,

$$
H^{G}(t, \mathbf{r})=\left[\begin{array}{lll}
\phi_{1}(\mathbf{r}) & \ldots & \phi_{K}(\mathbf{r})
\end{array}\right] B(t)+\bar{\xi}_{\text {non-tidal }}(\mathbf{r}) .
$$

The filtering equations for parameter estimation are given by

$$
\begin{aligned}
G^{(-)}(t) & =G(t-1) \\
P^{G(-)}(t) & =P^{G}(t-1)+W^{G}(t-1) \\
K^{G} & =P^{G(-)}(t)\left(H^{G}\right)^{T}\left(H^{G} P^{G(-)}(t)\left(H^{G}\right)^{T}+R^{\text {depth }}\right)^{-1} \\
G(t) & =G^{(-)}(t)+K^{G}\left(z^{\text {depth }}(t, \mathbf{r})-M^{G}-H^{G} G^{(-)}(t)\right) \\
P^{G}(t) & =\left(I-K^{G} H^{G}\right) P^{G(-)}(t)\left(I-K^{G} H^{G}\right)^{T}+K^{G} R^{\text {depth }}\left(K^{G}\right)^{T} .
\end{aligned}
$$



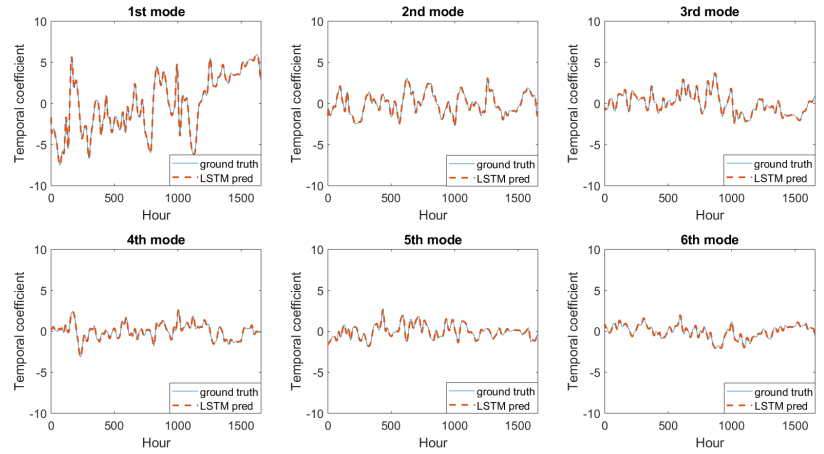

Figure 3: Comparison between ground truth temporal coefficients used for training the network, and the LSTM predicted temporal coefficients for the W-E direction flow field.

\section{SIMULATION RESULTS}

In this section, the simulation results of using LSTM data assimilation methods are presented. First, we will show the results of using LSTM to predict temporal coefficients. Then we will present the results of using LSTM based Kalman Filter for data assimilation of the ocean flow field.

The HFR data input is given by an HFR product made available by Dana Savidge (Skidaway Institute of Oceanography, University of Georgia) and Sara Haines (University of North Carolina at Chapel Hill) that combines radials from two different HFR systems installed near Cape Hatteras, NC. The 2-D surface flow field is a least squares fit to a combination of radial velocities from a long range Coastal Ocean Dynamics Applications Radar (CODAR) and a nested high resolution Wellen Radar (WERA), with an approximate 5 - $6 \mathrm{~km}$ horizontal resolution.

In this simulation experiment, we train the LSTM with 1657 historical data points, and then test its performance with another 199 data points. A single hidden layer LSTM network with 100 hidden units is used for state prediction. We use the ground truth coefficients time-delayed vector as input for the LSTM. The choice of time-delayed parameter $N$, which is 3 in this simulation, is based on the time window $T_{L}$ of Jacobian matrix.

Through the EOF analysis, the spatial and temporal varying flow field can be decomposed into the time-averaged field, and the six EOF modes multiplied with their corresponding time coefficients. As shown in Figure 3 and Figure 4, compared with the ground truth of the temporal coefficients, the LSTM network is able to generate accurate predictions for the training dataset of temporal coefficients of EOF modes. The LSTM predictions reveal the same trend as the ground truth and have root mean square errors less than 0.005 which shows that the predicted temporal coefficients have little difference from the ground truth. After the LSTM network is trained, we can test the network using testing dataset. As shown in Figure 5 and Figure 6, the trained LSTM is also capable of making accurate prediction for the testing dataset compared with the ground truth The temporal evolution of the flow field is well captured by the trained LSTM network, both in short term and in relatively long term. Table 1 demonstrates LSTM's capability of making satisfying prediction with low root mean square error.
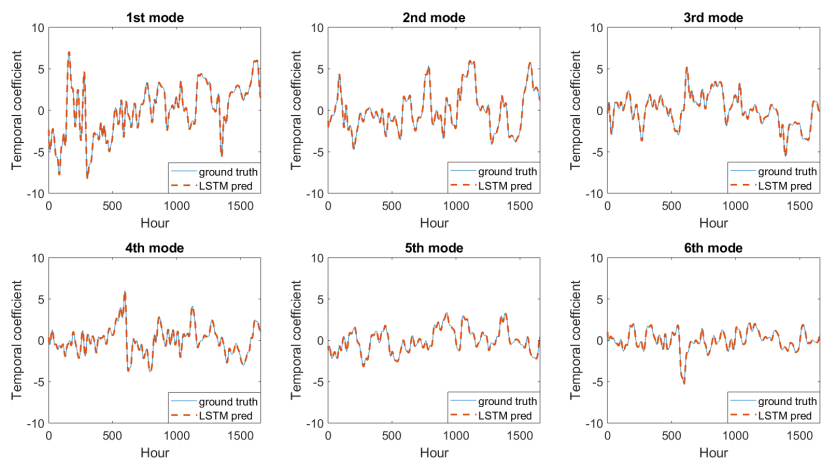

Figure 4: Comparison between ground truth temporal coefficients used for training the network, and the LSTM predicted temporal coefficients for the N-S direction flow field.

Table 1: RMSE between ground truth temporal coefficients used for testing the network, and the LSTM predicted temporal coefficients for $\mathrm{W}$-E and $\mathrm{N}-\mathrm{S}$ direction flow field.

\begin{tabular}{lcl}
\hline & W-E direction & N-S direction \\
\hline 1st mode & 0.0205 & 0.0110 \\
\hline 2nd mode & 0.0112 & 0.0244 \\
\hline 3rd mode & 0.0298 & 0.0342 \\
\hline 4th mode & 0.0187 & 0.0207 \\
\hline 5th mode & 0.0078 & 0.0079 \\
\hline 6th mode & 0.0061 & 0.0063
\end{tabular}
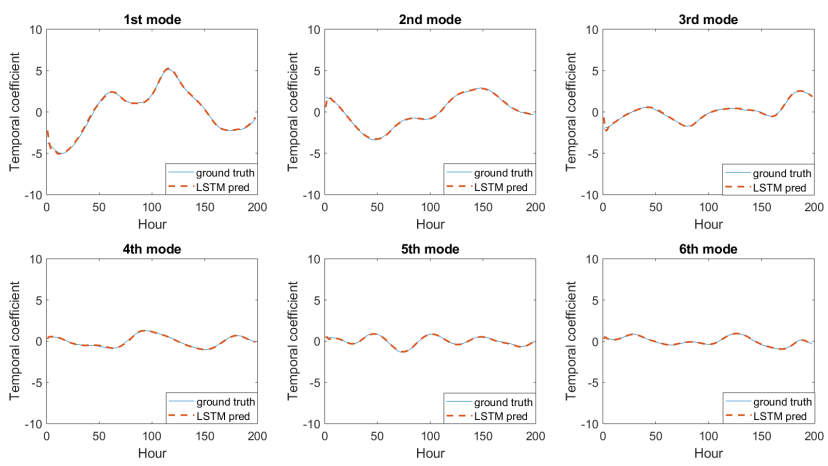

Figure 5: Comparison between ground truth temporal coefficients used for testing the network, and the LSTM predicted temporal coefficients for the W-E direction flow field.

Given the set of temporal coefficient predicted by the LSTM, the flow speed prediction from the Kalman Filter can be computed. As shown in Figure 7, we compare the one time step ahead flow prediction from the LSTM based Kalman Filter with the glider derived flow estimation and HF Radar reported surface flow measurement along glider trajectory. From the plot, it is shown that the LSTM-Kalman 

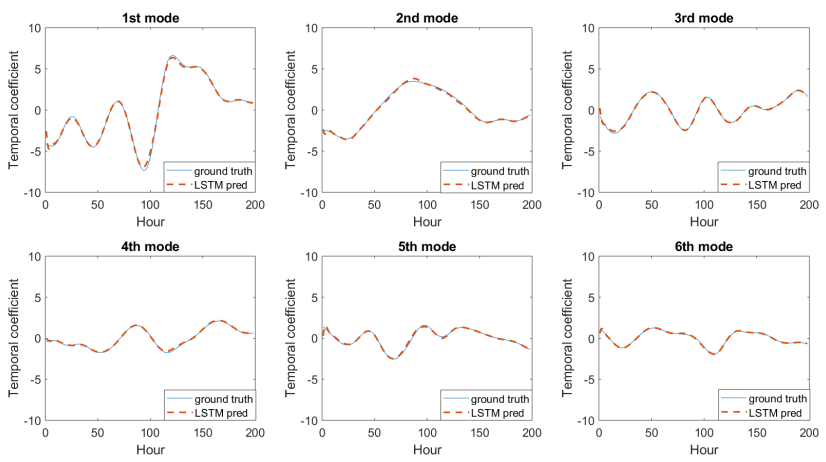

Figure 6: Comparison between ground truth temporal coefficients used for testing the network, and the LSTM predicted temporal coefficients for the N-S direction flow field.
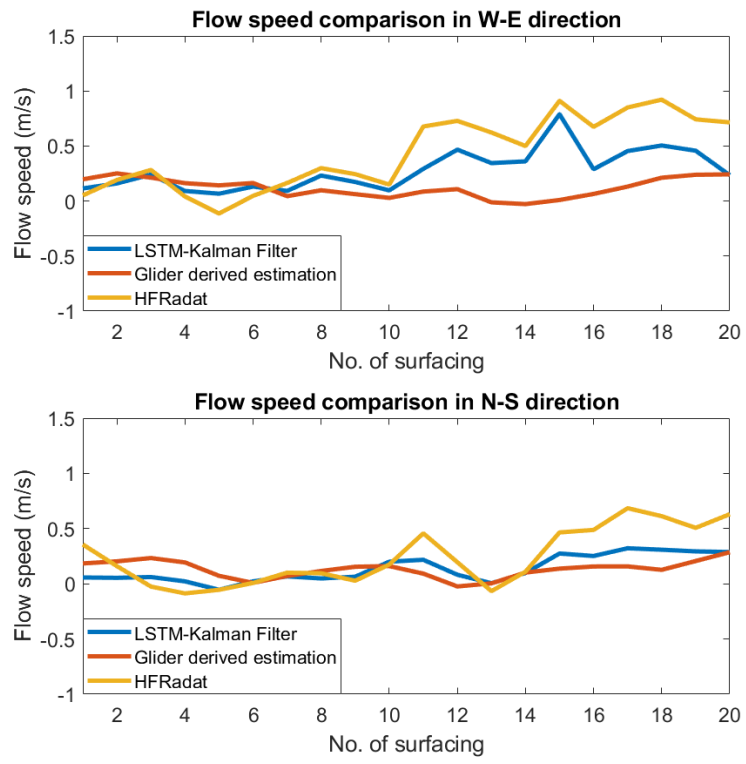

Figure 7: Comparison between glider derived flow estimation, HF Radar reported surface flow measurement, and the flow estimation from LSTM based Kalman Filter along glider trajectory in 20 consecutive diving-surfacing events.

Filter prediction reports a similar trend as the glider-estimated flow and the HFR measurement.

We also estimate surface to depth-averaged flow conversion rate. As shown in Figure 8, value of the $G$ function fluctuates over time. The $\mathrm{G}$ function of $\mathrm{W}-\mathrm{E}$ direction flow has significant deviation from 1 at the $15^{\text {th }}$ surfacing, indicating that the estimated depth averaged flow has significant difference from the surface flow. As shown in Figure 7 , at the $15^{t h}$ surfacing, the HFR surface flow measurement has significant difference from the depth-averaged glider derived flow estimation, which results in the fluctuation of the $\mathrm{G}$ function in W-E direction at the $15^{\text {th }}$ surfacing event. Similarly, for the $G$ parameter in N-S direction, it shows large variation at
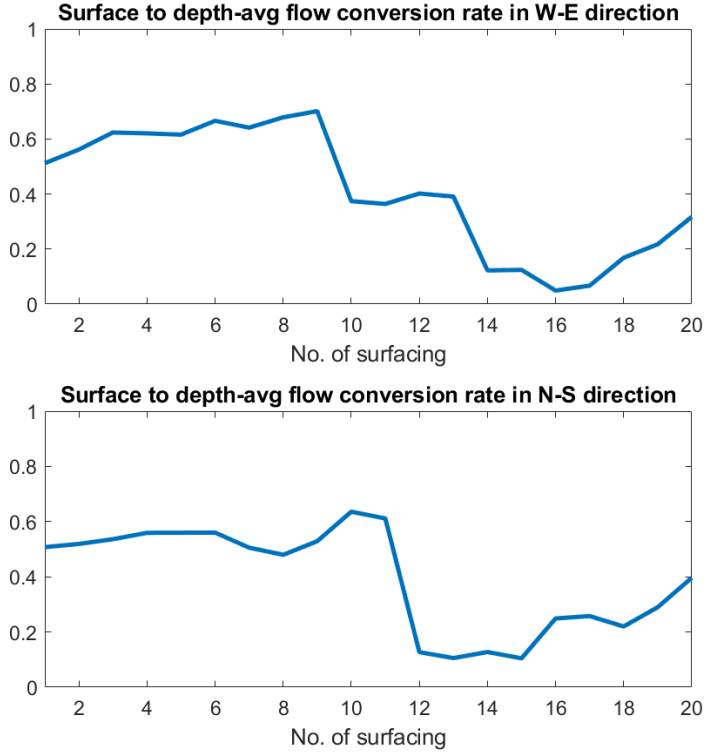

Figure 8: Estimated surface to depth averaged flow conversion rate in 20 diving-surfacing events.

the $17^{\text {th }}$ surfacing, which is approximately the time when there is large difference between the glider estimated flow and the HFR measurement, as shown in Figure 7.

\section{CONCLUSIONS}

In this paper, we present an LSTM-based Kalman Filter for data assimilation of a spatio-temporally varying depth-averaged ocean flow field for underwater glider path planning. The Kalman Filter is performed to combine the dynamics derived from the LSTM network, and the observations from the glider flow estimation data. Simulation results demonstrate that the proposed data assimilation method can give flow field prediction of accuracy.

\section{ACKNOWLEDGMENTS}

The research work is supported by ONR grants N00014-19-1-2266 and N00014-16-1-2667; NSF grants OCE-1559475, CNS-1828678, and S\&AS-1849228; NRL grants N0017317-1-G001 and N00173-19P-1412 ; and NOAA NA16NOS0120028.

\section{REFERENCES}

[1] Laurent Bertino, Geir Evensen, and Hans Wackernagel. 2003. Sequential data assimilation techniques in oceanography. International Statistical Review 71, 2 (2003), 223-241.

[2] Mark A Cane, Alexey Kaplan, Robert N Miller, Benyang Tang, Eric C Hackert, and Anthony J Busalacchi. 1996. Mapping tropical Pacific sea level: Data assimilation via a reduced state space Kalman filter. Journal of Geophysical Research: Oceans 101, C10 (1996), 22599-22617.

[3] Eric P. Chassignet, Harley E. Hurlburt, Ole Martin Smedstad, George R. Halliwell, Patrick J. Hogan, Alan J. Wallcraft, Remy Baraille, and Rainer Bleck. 2007. The HYCOM (HYbrid Coordinate Ocean Model) data assimilative system. Fournal of Marine Systems 65, 1 (2007), $60-83$.

[4] Huseyin Coskun, Felix Achilles, Robert DiPietro, Nassir Navab, and Federico Tombari. 2017. Long short-term memory kalman filters: Recurrent neural estimators for pose regularization. In Proceedings of the IEEE International Conference 
on Computer Vision. 5524-5532.

[5] Emanuele Di Lorenzo, Andrew M Moore, Hernan G Arango, Bruce D Cornuelle Arthur J Miller, Brian Powell, Boon S Chua, and Andrew F Bennett. 2007. Weak and strong constraint data assimilation in the inverse Regional Ocean Modeling System (ROMS): Development and application for a baroclinic coastal upwelling system. Ocean Modelling 16, 3-4 (2007), 160-187.

[6] Charles C. Eriksen, T. James Osse, Russell D. Light, Timothy Wen, Thomas W. Lehman, Peter L. Sabin, John W. Ballard, and Andrew M. Chiodi. 2001. Seaglider: A Long-Range Autonomous Underwater Vehicle for Oceanographic Research. IEEE Journal of Oceanic Engineering 26, 4 (2001), 424-436.

[7] Annalisa Griffa, Leonid I. Piterbarg, and Tamay Ozgokmen. 2004. Predictability of Lagrangian particle trajectories: Effects of smoothing of the underlying Eulerian flow. Fournal of Marine Research 62, 1 (2004), 1-35.

[8] D.B. Haidvogel, H. Arango, W.P. Budgell, B.D. Cornuelle, E. Curchitser, E. Di Lorenzo, K. Fennel, W.R. Geyer, A.J. Hermann, L. Lanerolle, J. Levin, J.C. McWilliams, A.J. Miller, A.M. Moore, T.M. Powell, A.F. Shchepetkin, C.R. Sherwood, R.P. Signell, J.C. Warner, and J. Wilkin. 2008. Ocean forecasting in terrainfollowing coordinates: Formulation and skill assessment of the Regional Ocean Modeling System. 7. Comput. Phys. 227, 7 (2008), 3595 - 3624.

[9] Angelique C. Haza, Leonid I. Piterbarg, Paul Martin, Tamay M. Ozgokmen, and Annalisa Griffa. 2007. A Lagrangian subgridscale model for particle transport improvement and application in the Adriatic Sea using the Navy Coastal Ocean Model. Ocean Modelling 17, 1 (2007), 68-91.

[10] Sepp Hochreiter and JẪijrgen Schmidhuber. 1997. Long Short-Term Memory. Neural Computation 9, 8 (1997), 1735-1780. https://doi.org/10.1162/neco.1997.9. 8.1735

[11] Naomi E. Leonard, Derek A. Paley, Russ E. Davis, David M. Fratantoni, Francois Lekien, and Fumin Zhang. 2010. Coordinated Control of an Underwater Glider Fleet in an Adaptive Ocean Sampling Field Experiment in Monterey Bay. fournal of Field Robotics 27, 6 (2010), 718-740.

[12] Zhijin Li, Yi Chao, James C McWilliams, and Kayo Ide. 2008. A three-dimensional variational data assimilation scheme for the regional ocean modeling system. Journal of Atmospheric and Oceanic Technology 25, 11 (2008), 2074-2090.

[13] Yan Liang, De Xi An, Dong Hua Zhou, and Quan Pan. 2004. A finite-horizon adaptive Kalman filter for linear systems with unknown disturbances. Signal Processing 84, 11 (2004), 2175 - 2194. https://doi.org/10.1016/j.sigpro.2004.06.021 Special Section Signal Processing in Communications.

[14] Edward N Lorenz. 1956. Empirical orthogonal functions and statistical weather prediction. Technical Report.

[15] K. Lynch, I. Schwartz, P. Yang, and R. Freeman. 2008. Decentralized environmenta modeling by mobile sensor networks. In IEEE Transaction of Robotics, Vol. 24 710-724.

[16] R. Mehra. 1972. Approaches to adaptive filtering. IEEE Transactions on Automatic Control 17, 5 (October 1972), 693-698. https://doi.org/10.1109/TAC.1972.1100100

[17] Paritosh Mokhasi, Dietmar Rempfer, and Sriharsha Kandala. 2009. Predictive flow-field estimation. Physica D: Nonlinear Phenomena 238, 3 (2009), 290-308.

[18] Said Ouala, Ronan Fablet, Cédric Herzet, Bertrand Chapron, Ananda Pascual, Fabrice Collard, and Lucile Gaultier. 2018. Neural Network Based Kalman Filters for the Spatio-Temporal Interpolation of Satellite-Derived Sea Surface Temperature Remote Sensing 10, 12 (2018), 1864.

[19] Jr. R. A .Luettich, J. J. Westerink, and Norman W. Scheffner. 1992. ADCIRC: AN AD VANCED THREE-DIMENSIONAL CIRCULATION MODEL FOR SHELVES, COASTS, AND ESTUARIES. Technical Report NRL/FR/7322-00-9962. Naval Research Lab.

[20] Daniel Rudnick, Daniel Costa, Ken Johnson, Craig Lee, and Mary-Louise Timmermans. 2018. ALPS II - Autonomous Lagrangian Platforms and Sensors. A Report of the ALPS II Workshop. Technical Report. 66 pages.

[21] Samuele Salti and Luigi Di Stefano. 2013. On-line Support Vector Regression of the transition model for the Kalman filter. Image and Vision Computing 31, 6 (2013), 487 - 501. https://doi.org/10.1016/j.imavis.2012.09.008 Machine learning in motion analysis: New advances.

[22] Alexander F. Shchepetkin and James C. McWilliams. 2005. The regional oceanic modeling system (ROMS): a split-explicit, free-surface, topography-followingcoordinate oceanic model. Ocean Modelling 9, 4 (2005), 347 - 404.

[23] J. Sherman, R. E. Davis, W. B. Owens, and J. A. Valdes. 2001. The autonomous underwater glider "Spray". IEEE Journal of Oceanic Engineering 26, 4 (2001), 437-446.

[24] Ryan N. Smith, Yi Chao, Peggy P. Li, David A. Caron, Burton H. Jones, and Gaurav S. Sukhatme. 2010. Planning and Implementing Trajectories for Autonomous Underwater Vehicles to Track Evolving Ocean Processes Based on Predictions from a Regional Ocean Model. The International fournal of Robotics Research 29, 12 (2010), 1475-1497.

25] H. Stommel. 1989. The Slocum Mission. Oceanography 2 (1989), 22-25.

[26] K. Szwaykowska and F. Zhang. 2014. Trend and Bounds for Error Growth in Controlled Lagrangian Particle Tracking. IEEE fournal of Oceanic Engineering 39, 1 (January 2014), 10-25

[27] D. C. Webb, P. J. Simonetti, and C. P. Jones. 2001. SLOCUM: an underwater glider propelled by environmental energy. IEEE fournal of Oceanic Engineering 26, 4
(2001), 447-452.

[28] Fumin Zhang, D. M. Fratantoni, D. A. Paley, J. M. Lund, and N. E. Leonard. 2007. Control of coordinated patterns for ocean sampling. Internat. F. Control 80, 7 (2007), 1186-1199. 